\title{
What Factors Predict Failure of Nonsurgical Management of a Lumbar Surgical Site Infection?
}

\author{
CHRISTOPHER J. LUCASTI, BS, ${ }^{1,2}$ MYLES DWORKIN, BS, ${ }^{1,2}$ KRIS E. RADCLIFF, MD, ${ }^{2}$ \\ KRISTEN NICHOLSON, PHD,${ }^{2}$ CHRISTOPHER J. LUCASTI, DO ${ }^{3}$ BARRETT I. WOODS, MD $^{2}$ \\ ${ }^{I}$ Sidney Kimmel Medical College at Thomas Jefferson University, Philadelphia, Pennsylvania, ${ }^{2}$ Department of Orthopaedic Surgery, Thomas Jefferson University \\ and The Rothman Institute, Philadelphia, Pennsylvania, ${ }^{3}$ South Jersey Infectious Disease, Somers Point, New Jersey
}

\begin{abstract}
Background: One of the most frequent complications of invasive lumbar spine surgery is postoperative surgical site infections (SSIs). Although there are absolute criteria for surgical intervention (progressive neurologic deficit, sepsis, failure of medical management), the treatment of routine, uncomplicated SSIs remains somewhat unclear. The purpose of this study was to evaluate the outcome of a series of patients with postoperative surgical site infections who were treated with or without surgical intervention. The primary clinical outcome was the assessment of whether medical management alone would be sufficient to eradicate the infection.

Methods: A retrospective review of consecutive patients who underwent lumbar surgery complicated by spine infection between 2011 and 2017 was performed in order to determine what factors, if any, resulted in the need for additional surgical management. Medical records were reviewed for various demographic (e.g., age), clinical (e.g., organism), and surgical (e.g., presence of instrumentation) factors. A regression analysis was performed to identify what variables significantly increased the risk for SSI.

Results: During the 6-year period studied, a total of 74 patients met the inclusion criteria and were included in the study. There were 13 patients who failed medical management and required additional surgical management, which included irrigation and debridement. Thus, overall, medical management alone was effective in $82 \%$ of patients. In the final multivariate logistic regression analysis model, revision primary surgery had the strongest association with SSI that would require a washout. In addition, diabetes had a strong association with the occurrence of an infection.

Conclusions: Identification of risk factors associated with the need for additional surgical management may benefit from aggressive antibiotic therapy to reduce the likelihood of reoperation. Clinicians should be aware of the identified risk factors, which may help with postoperative management in at-risk individuals
\end{abstract}

Lumbar Spine

Keywords: infection, irrigation and debridement, lumbar surgery, nonsurgical management, SSI

\section{INTRODUCTION}

Spine surgery is one of the most common surgical procedures performed in the United States, with approximately 1 million cases in $2011 .^{1}$ This number has increased rapidly during the past decade and is predicted to continue to rise. ${ }^{1}$ Spine surgery is indicated for a number of diagnoses, including fractures or dislocations, degeneration, spinal cord injuries, congenital anomalies, and inflammatory spondylopathy. ${ }^{2}$ One of the most serious complications of spine surgery is postoperative surgical site infections $(\mathrm{SSI}){ }^{3}$ The literature reports that rates following spine surgery may range from $0.7 \%$ to $11.9 \%$ depending on factors such as surgical approach, instrumentation use, preoperative symptoms, intervertebral levels effected, and patient demographics. $^{4-7}$ A number of variables have been shown to increase the risk of SSI following spine surgery, including obesity, drug or alcohol use, and diabetes. ${ }^{8}$ Risk factors should be minimized preoperatively in order to lower incident rates. ${ }^{9}$

Spinal SSIs have far-reaching impacts on both patient outcomes and health care economics. Direct costs, such as longer hospital stay; frequent procedures, including irrigation and debridement; and additional radiologic tests can add a significant financial burden to all parties involved. ${ }^{10} \mathrm{~A}$ single infection may cost an additional $\$ 15800$ to $\$ 43900$, which is 4 times greater than the initial surgery. ${ }^{11}$ During the course of a year this may lead to hospital systems paying more than $\$ 2000000 .{ }^{12}$ SSIs also greatly impact patient recovery. They have been shown to increase length of hospital stay by more than 5 days and readmission rates by $600 \% .{ }^{12}$ SSIs 
may also reduce quality of life and prolong rehabilitation. ${ }^{13}$ Furthermore, treatment of SSIs is difficult and complicated, especially in those patients with instrumentation present. ${ }^{14}$

SSIs may be treated surgically or medically. The absolute criteria for surgical intervention include progressive neurologic deficit, sepsis, and failure of medical management. ${ }^{15}$ In the absence of these indicators, however, the treatment of routine, uncomplicated SSIs remains unclear. Revision surgery is associated with risk of perioperative anesthesia complications, increased pain, and additional infections. ${ }^{16}$ Surgery, however, can avert sepsis and the development of a permanent neurologic deficit. ${ }^{15}$ Medical management with antimicrobial agents or negative pressure dressings may be preferred for uncomplicated superficial SSIs, but this risks treatment failure. ${ }^{17}$ The clinical distinction between surgical and medical management has not been well defined. The purpose of this study is to evaluate the outcome of a series of patients with postoperative lumbar SSIs who were treated with or without surgical intervention.

\section{METHODS}

A retrospective review of consecutive patients who underwent lumbar surgery complicated by spine infection between 2011 and 2017 was performed in order to determine what factors, if any, resulted in the need for additional surgical management. During this interval, one author (an infectious disease specialist) was responsible for the medical management of the infections. Inclusion criteria were patients 18 and older at the time of surgery who had a proven SSI in the lumbar spine. The analysis excluded patients who underwent cervical $(n=13)$ or thoracic $(n=2)$ surgery, as well as patients with less than 30 days of follow-up data (n $=6$ ). Patients with less than 30 days of follow-up data were excluded from the study because of the inability to determine whether the infection was cleared or if the patient followed up with a practitioner outside of the study.

Medical records were reviewed for various demographic (e.g., age, sex), clinical (e.g., organism), and surgical (e.g., presence of instrumentation) factors. The primary clinical outcome was the assessment of whether medical management alone would be sufficient to eradicate the infection, which was determined based on follow-up laboratory data. In this study, revision surgery was defined as a patient requiring removal of hardware for an unsuccessful previous surgery done at an outside hospital. The statistical computing environment $\mathrm{R}$ was used to perform multivariate logistic regression model to identify independent risk factors for failure of medical management.

\section{RESULTS}

During the 6-year period studied, a total of 74 patients met the inclusion criteria and were included in the study. The average age of the cohort was 53.3 years; 47 patients $(63.5 \%)$ were male and $27(36.5 \%)$ were female. The average body mass index (BMI) of the entire study cohort was 30.74. A total of 17 patients $(22.9 \%)$ were smokers and $14(18.9 \%)$ were diabetic. The average time from the operation to the diagnosis of the infection was 20.0 days. Two infections could be classified as late infections because they occurred $>90$ days after the index surgery.

The most common microorganism isolated from the cultures obtained from the surgical wounds was Staphylococcus aureus ( $S$ aureus), which was found in $51.3 \%$ of the cases. Other cultured microorganisms were Pseudomonas, Escherichia coli, Streptococcus, and coagulase-negative $S$ aureus.

A total of 22 patients $(29.7 \%)$ were treated with a wound vacuum assisted closure (VAC) during their management. The average white blood cell (WBC) count, C-reactive protein (CRP), and erythrocyte sedimentation rate (ESR) on presentation were 8.6 $\mu \mathrm{L}, 22.9$, and 41.8 , respectively.

There were 13 patients that failed medical management and required additional surgical management, which included irrigation and debridement (I\&D). Thus, overall, medical management alone was effective in $82 \%$ of patients. The average age of these patients who required a second surgery was 47 \pm 17 years. There were 11 women $(84.6 \%)$ who required I\&D compared with 2 men $(15.4 \%)$ who required I\&D. The average BMI of these patients was 32.9. A total of 5 of the patients requiring I\&D were smokers $(38.4 \%)$, and $4(30.7 \%)$ were diabetic. A total of 6 of the 13 patients who had a secondary surgery $(46.1 \%)$ were treated first with a VAC prior to undergoing surgical management. The average WBC count, CRP, and ESR of those patients requiring $\mathrm{I} \& \mathrm{D}$ were $7.6 \mu \mathrm{L}, 16.5$, and 28 , respectively.

There were 61 patients who did not fail medical management, and their infections were resolved 
Table 1. Multivariate logistic regression model identifying independent risk factors for failure of medical management.

\begin{tabular}{|c|c|c|c|c|}
\hline & $\mathbf{N}$ & $\mathbf{Y}$ & Univariable Logistic & Wald $\chi^{2}$ Statistic \\
\hline Age, mean (SD) & $50(15)$ & $47(17)$ & 0.500 & 0.502 \\
\hline BMI, median [IQR] & $28.4[25.8,33.3]$ & $32.9[29.3,37.1]$ & 0.401 & 0.405 \\
\hline WBC, median [IQR] & $8.5[6.9,9.3]$ & $7.6[6.4,8.9]$ & 0.764 & 0.765 \\
\hline CRP, median [IQR] & $3.3[0.7,10.3]$ & $16.5[6.7,56.0]$ & 0.034 & 0.037 \\
\hline ESR, median [IQR] & $31[14,54]$ & $28[27,70]$ & 0.520 & 0.522 \\
\hline \multicolumn{5}{|l|}{ Obese } \\
\hline$<30$ & 27 & 4 & 0.229 & 0.233 \\
\hline $30+$ & 24 & 8 & & \\
\hline \multicolumn{5}{|l|}{ Sex } \\
\hline M (0) & 25 & 2 & 0.099 & 0.103 \\
\hline F (1) & 36 & 11 & & \\
\hline \multicolumn{5}{|l|}{ Smoking status } \\
\hline $\mathrm{N}$ & 48 & 8 & 0.162 & 0.166 \\
\hline $\mathrm{Y}$ & 12 & 5 & & \\
\hline \multicolumn{5}{|l|}{ Diabetic } \\
\hline $\mathrm{N}$ & 51 & 9 & 0.238 & 0.242 \\
\hline $\mathrm{Y}$ & 10 & 4 & & \\
\hline \multicolumn{5}{|l|}{ Trauma } \\
\hline $\mathrm{N}$ & 36 & 8 & 0.866 & 0.867 \\
\hline $\mathrm{Y}$ & 25 & 5 & & \\
\hline \multicolumn{5}{|l|}{ CVD } \\
\hline $\mathrm{N}$ & 56 & 13 & 0.993 & 0.993 \\
\hline $\mathrm{Y}$ & 5 & 0 & & \\
\hline \multicolumn{5}{|l|}{ HTN } \\
\hline $\mathrm{N}$ & 35 & 8 & 0.783 & 0.783 \\
\hline $\mathrm{Y}$ & 26 & 5 & & \\
\hline \multicolumn{5}{|l|}{$\mathrm{HCV}$} \\
\hline $\mathrm{N}$ & 58 & 13 & 0.991 & 0.991 \\
\hline $\mathrm{Y}$ & 3 & 0 & & \\
\hline \multicolumn{5}{|l|}{ HIV } \\
\hline $\mathrm{N}$ & 60 & 13 & 0.992 & 0.992 \\
\hline $\mathrm{Y}$ & 1 & 0 & & \\
\hline \multicolumn{5}{|l|}{ VAC } \\
\hline $\mathrm{N}$ & 45 & 7 & 0.161 & 0.165 \\
\hline Y & 16 & 6 & & \\
\hline \multicolumn{5}{|l|}{ Discharge } \\
\hline Home & 55 & 11 & 0.562 & 0.564 \\
\hline Rehabilitation & 6 & 2 & & \\
\hline \multicolumn{5}{|l|}{ Revision } \\
\hline $\mathrm{N}$ & 59 & 12 & 0.477 & 0.479 \\
\hline $\mathrm{Y}$ & 3 & 1 & & \\
\hline \multicolumn{5}{|l|}{ IR aspiration } \\
\hline $\mathrm{N}$ & 57 & 13 & 0.994 & 0.994 \\
\hline $\mathrm{Y}$ & 4 & 0 & & \\
\hline
\end{tabular}

Abbreviations: BMI, body mass index; CRP, C-reactive protein; CVD, cardiovascular disease; ESR, erythrocyte sedimentation rate; HCV, hepatitis C virus; HIV, human immunodeficiency virus; HTN, hypertension; IQR, interquartile range; IR, interventional radiology; VAC, wound vacuum assisted closure; WBC, white blood cell.

without the need for further surgical intervention. The average age of these patients was $50 \pm 15$ years $(P=.045)$. There were 36 women $(59.0 \%)$ and 25 men $(41.0 \% ; P=.038)$. The average BMI of these patients was $28.4(P=.796)$. A total of 12 of the patients medically managed were smokers $(19.7 \%$; $P=.129)$ and 10 were diabetic $(16.4 \% ; P=.008)$. Sixteen of the patients $(26.2 \%)$ were treated with a VAC during their management $(P=.091)$. The average WBC count, CRP, and ESR of those patients who were medically managed were $8.5 \mu \mathrm{L}$, 3.3 , and 31 , respectively $(P=.665 ; P=.037 ; P=$ $.180)$.

The results of the multivariate logistic regression model to identify independent risk factors for failure of medical management are listed in Table 1. In the final model, revision primary surgery had the strongest association with SSI that would require a washout, with an odds ratio (OR) of 63.52. In addition, diabetes had a strong association with the occurrence of an infection, with an OR of 33.49. Other variables that had a strong association with SSI include female sex, OR 9.90; smokers, OR 5.21; use of a VAC, OR 4.90; CRP, OR 1.02; and age, OR 0.93.

\section{Regression Analysis}

Univariate analysis identified 6 variables initially (CRP, obesity, sex, smoking, diabetes, VAC) at the $0.25 \alpha$ level using the Wald $\chi^{2}$ statistic (Table 1). In the initial multivariate, obesity was removed be- 
Table 2. Final main effects model.

\begin{tabular}{lcc}
\hline & $\boldsymbol{P}$ Value & OR $\mathbf{( 9 5 \%}$ CI) \\
\hline CRP & .037 & $1.02(1.00,1.04)$ \\
Sex $=$ F & .038 & $9.90(1.46,129.35)$ \\
Smoking status $=$ Y & .129 & $5.21(0.65,53.17)$ \\
Diabetic = Y & .008 & $33.49(3.22,647.53)$ \\
VAC $=$ Y & .091 & $4.90(0.86,39.49)$ \\
Age & .045 & $0.93(0.86,0.99)$ \\
Revision $=$ Y & .046 & $63.52(1.14,6412.98)$ \\
\hline
\end{tabular}

Abbreviations: CI, confidence interval; CRP, C-reactive protein; OR, odds ratio; VAC, wound vacuum assisted closure.

cause it was not significant $(P=.430)$, and its removal did not change any remaining parameter estimates by more than $20 \%$. Although VAC use was not significant at the $0.15 \propto$ level $(P=.192)$, removing this variable did change the parameter estimate for smoking by $23 \%$, so it was retained. The remaining variables (CRP $P=.082$; sex $P=$ .101 ; smoking $P=.082$; diabetic $P=.018$ ) were all retained for being significant at the $\alpha=0.15$ level.

Of the variables that were not initially significant at the 0.25 level, only age and revision were included back in the model when tested with the 5 retained covariates; both were significant at the $0.15 \alpha$ level and both changed parameter estimates by more than $20 \%$. The final multivariable model included CRP, sex, smoking status, diabetes, VAC, age at index procedure, and revision (Table 2).

Of the retained variables, only age and diabetes had a significant association. Diabetic patients had a mean age of 14 years older than nondiabetic patients. The variable inflation factor (VIF) values for our final model had a range of 1.4 to 2.2, suggesting multicollinearity does not have a considerable effect on our variable selection in the final model.

Backward stepwise regression for a full model was not possible without an initial paring of variables. There were too many exploratory variables for the sample size; an attempt to run such a model did not converge. Forward stepwise regression yielded CRP, sex, smoking status, diabetes, age at index, and radiologic aspiration. It did not include revision or VAC use. The radiologic aspiration variable was not retained in our purposeful selection model but was retained in the forward selection and had a $P$ value of .995 and an OR $<0.001$. In both the forward selection and purposeful selection models, age had an odds ratio of $<1$. Excluding the aforementioned radiologic aspiration variable, the remaining variables in both models had ORs $>1$. Not unexpectedly, there are small differences in the final model with the 2 approaches to variable selection. However, the forward selection produced an almost nonsensical result for radiologic aspiration (OR of almost 0 with an associated $P=.995)$. Moreover, the VIF values from this model ranged upwards of 3.4. Although a value of 3.4 for VIF is not a concerning value for multicollinearity, when compared to smaller VIF values in our model, we feel even more confident in our choice of purposeful selection for this particular study.

\section{DISCUSSION}

Overall, most patients (82\%) with SSIs were successfully treated with medical management. This study successfully identified risk factors found among the cohort of patients who failed medical management and required surgical intervention. The most significant risk factor identified was presenting for surgery because of the revision of a prior procedure. Patients with diabetes and women also had a higher risk of requiring surgical management to resolve infections. Additionally, the most common organism cultured from the infected wounds was methicillin-sensitive $S$ aureus, and the average time from the index surgery to diagnosis of infection was 20 days.

Although there are a multitude of factors associated with an increased risk of SSIs, the literature is scarce with regard to which patients may fail medical management following the development of an infection. Our study found that patients undergoing revision surgery were most at risk of failure. This corresponds to previous findings that suggest revision surgery is a strong risk factor for developing an SSI following a lumbar procedure. $^{18,19}$ Smoking and diabetes have also been previously identified as risk factors. This study, however, is the first to predict failure of medical management based on these variables.

Additionally, our study identified the use of VACs to be associated with failure of medical management. Although I\&D is considered the standard of therapy for SSIs, VACs have been used for postoperative spine infection. These have proven to be an effective add-on therapy following I\&D to assist with wound closure. ${ }^{20}$ Although VACs have decreased wound complications, improved healing times, and reduced overall morbidity rates, their use may be a predictive factor for further surgical management. ${ }^{21}$ Patients who require a VAC for 
wound closure should be carefully monitored because they may be at a greater risk for the need for additional surgical intervention. Furthermore, other studies have demonstrated that the institution of prompt antibiotic therapy coupled with the use of VACs in patients with identified risk factors for developing an SSI may reduce the duration of treatment, eliminate the need for I\&D, decrease the length of hospital stay, and reduce health care expenses. ${ }^{20}$ Our findings support these claims and distinguish the use of VACs as a risk factor for failed medical management.

It is important for the clinician to understand the importance of early recognition and prompt administration of antibiotics as well as how to recognize patients who may require multiple I\&Ds or more complex management. Previously, Dipaola et al. ${ }^{22}$ described the PITTS score to determine what patient factors predict the need for complicated SSI management following spine surgery. The most significant findings were patients with methicillinresistant Staphylococcus aureus cultures and those with distant site infections or bacteremia. Other predictors include instrumentation, surgery to the posterior lumbar spine, use of nonautograft bone graft material, and diabetes. Recently, Lee et al. ${ }^{23}$ developed an algorithm that uses preoperative risk factors to predict the development of an SSI following spine surgery. It provides an absolute percent likelihood of an SSI, which can be used in patient consultations. We suggest that use of the PITTS score and Lee algorithm to help clinicians determine which patients are at increased risk of developing an SSI following spine surgery as medical management is more successful if early diagnosis is made. ${ }^{24}$ We recommend early administration of antibiotics if there is a strong suspicion of infection. Failure to eliminate SSIs has been associated with a significantly greater morbidity, mortality, and health care expenditure. ${ }^{10-13}$ The authors suggest that patients with risk factors such as older age, female sex, diabetes, and smoking may need closer follow-up because they are at risk of failure of medical management, which can result in increased health care expenditure.

A weakness of this study is the relatively small sample size of infected patients $(\mathrm{n}=74)$ and those requiring additional surgical management $(n=13)$. This is likely due to the use of a small private practice rather than a large institution. The practice setting (a community infectious disease practice) also likely increased the rate of follow-up. Furthermore, although precise clinical management of SSIs varies with clinician, it should be noted that our protocol does differ from some examples in the literature. Many studies included the use of I\&D as part of their initial treatment regimen, which differs from our protocol. ${ }^{10,14}$ This protocol was established in order to minimize additional procedures.

\section{CONCLUSION}

In conclusion, our study identified several risk factors for the failure of medical management following the development of an SSI in patients undergoing lumbar surgical procedures. The most important factors include revision surgery, diabetes, and female sex. These patients warrant additional monitoring and are more likely to require surgical treatment. To the author's knowledge this is the first study to identify risk factors associated with an increased likelihood of failure of medical management of an SSI following lumbar surgery. Additional studies with larger sample sizes should be conducted in order to confirm findings.

\section{REFERENCES}

1. Weiss AJ, Elixhauser A. Trends in operating room procedures in U.S. hospitals, 2001-2011. Statistical Brief 171. https://www.hcup-us.ahrq.gov/reports/statbriefs/sb171-Operat ing-Room-Procedure-Trends.jsp. Accessed January 18, 2019.

2. Lurie JD, Tosteson ANA, Deyo RA, Tosteson T, Weinstein J, Mirza SK. Indications for spine surgery: validation of an administrative coding algorithm to classify degenerative diagnoses. Spine (Phila Pa 1976). 2014;39(9):769-779.

3. Anderson DJ, Podgorny K, Berríos-Torres SI, et al. Strategies to prevent surgical site infections in acute care hospitals: 2014 update. Infect Control Hosp Epidemiol. 2014;35(6):605-627.

4. Abbey DM, Turner DM, Warson JS, Wirt TC, Scalley RD. Treatment of postoperative wound infections following spinal fusion with instrumentation. $J$ Spinal Disord. 1995;8(4):278-283.

5. Wang TY, Back AG, Hompe E, Wall K, Gottfried ON. Impact of surgical site infection and surgical debridement on lumbar arthrodesis: a single-institution analysis of incidence and risk factors. J Clin Neurosci. 2017;39:164-169.

6. Perry JW, Montgomerie JZ, Swank S, Gilmore DS, Maeder K. Wound infections following spinal fusion with posterior segmental spinal instrumentation. Clin Infect Dis. 1997;24(4):558-561.

7. Nagashima H, Tanishima S, Tanida A. Diagnosis and management of spinal infections. J Orthop Sci. 2018;23(1):8-13.

8. Saeedinia S, Nouri M, Azarhomayoun A, et al. The incidence and risk factors for surgical site infection after clean spinal operations: a prospective cohort study and review of the literature. Surg Neurol Int. 2015;6:154. 
9. Dobran M, Marini A, Nasi D, et al. Risk factors of surgical site infections in instrumented spine surgery. Surg Neurol Int. 2017;8:212.

10. Schimmel JJP, Horsting PP, de Kleuver M, Wonders G, van Limbeek J. Risk factors for deep surgical site infections after spinal fusion. Eur Spine J. 2010;19(10):1711-1719.

11. Dessy AM, Yuk FJ, Maniya AY, et al. Reduced surgical site infection rates following spine surgery using an enhanced prophylaxis protocol. Cureus. 2017;9(4):e1139.

12. Shepard J, Ward W, Milstone A, et al. Financial impact of surgical site infections on hospitals: the hospital management perspective. JAMA Surg. 2013;148(10):907-914.

13. Whitehouse JD, Friedman ND, Kirkland KB, Richardson WJ, Sexton DJ. The impact of surgical-site infections following orthopedic surgery at a community hospital and a university hospital: adverse quality of life, excess length of stay, and extra cost. Infect Control Hosp Epidemiol. 2002;23(4):183189.

14. Hegde V, Meredith DS, Kepler CK, Huang RC. Management of postoperative spinal infections. World $J$ Orthop. 2012;3(11):182-189.

15. Richmond BK, O'Brien B, Ubert A, Thompson S. Current treatment guidelines for postoperative surgical site infection: clinical considerations in the surgical care improvement project era. Am Surg. 2015;81(4):E179-E180.

16. Amlie E, Lerdal A, Gay CL, Høvik Ø, Nordsletten L, Dimmen S. A trend for increased risk of revision surgery due to deep infection following fast-track hip arthroplasty. $A d v$ Orthop. 2016;2016:7901953.

17. National Collaborating Centre for Women's and Children's Health (UK). Surgical Site Infection: Prevention and Treatment of Surgical Site Infection. London: RCOG Press; 2008. NICE Clinical Guidelines, No. 74: 7, postoperative phase. Available at: https://www.ncbi.nlm.nih.gov/books/NBK53739/. Accessed March 12, 2019.

18. Kurtz SM, Lau E, Ong KL, et al. Infection risk for primary and revision instrumented lumbar spine fusion in the Medicare population. J Neurosurg Spine. 2012;17(4):342-347.

19. Wilson J, Charlett A, Leong G, McDougall C, Duckworth G. Rates of surgical site infection after hip replacement as a hospital performance indicator: analysis of data from the English mandatory surveillance system. Infect Control Hosp Epidemiol. 2008;29(3):219-226.

20. Chen S, Anderson MV, Cheng WK, Wongworawat MD. Diabetes associated with increased surgical site infections in spinal arthrodesis. Clin Orthop Relat Res. 2009;467(7):1670 1673.

21. Kong L, Liu Z, Meng F, Shen Y. Smoking and risk of surgical site infection after spinal surgery: a systematic review and meta-analysis. Surg Infect. 2017;18(2):206-214.

22. Dipaola CP, Saravanja DD, Boriani L, et al. Postoperative infection treatment score for the spine (PITSS): construction and validation of a predictive model to define need for single versus multiple irrigation and debridement for spinal surgical site infection. Spine J. 2012;12(3):218-230.

23. Lee MJ, Cizik AM, Hamilton D, Chapman JR. Predicting surgical site infection after spine surgery: a validated model using a prospective surgical registry. Spine $J$. 2014;14(9):2112-2117.

24. Lee JS, Ahn DK, Chang BK, Lee JI. Treatment of surgical site infection in posterior lumbar interbody fusion. Asian Spine J. 2015;9(6):841-848.

Disclosures and COI: The authors received no funding for this study and report no conflicts of interest.

Corresponding Author: Christopher J. Lucasti, 2807 Sunset Ave, Longport, NJ 08403. Phone: (609) 350-3598; Email: Christopherjlucasti@gmail. com.

Published 30 June 2019

This manuscript is generously published free of charge by ISASS, the International Society for the Advancement of Spine Surgery. Copyright @ 2019 ISASS. To see more or order reprints or permissions, see http://ijssurgery.com. 